\begin{abstract}
Regulation of the dauBAR operon and characterization of $\mathrm{D}$-amino acid dehydrogenase DauA in arginine and lysine catabolism of Pseudomonas aeruginosa PAO1

Correspondence

Chung-Dar Lu

biocdl@langate.gsu.edu

Received 2 August 2009

Revised 8 October 2009

Accepted 19 October 2009

\section{Congran $\mathrm{Li}^{1}{ }^{1,2}$ Xiangyu $\mathrm{YaO}^{1}$ and Chung-Dar Lu ${ }^{1}$}

\author{
'Department of Biology, Georgia State University, Atlanta, GA 30303, USA \\ ${ }^{2}$ Laboratory of Pharmacology, Institute of Medicinal Biotechnology, Chinese Academy of Medical \\ Sciences and Peking Union Medical College, Beijing 100050, PR China
}

A unique D-to-L racemization of arginine by coupled arginine dehydrogenases DauA and DauB encoded by the dauBAR operon has been recently reported as a prerequisite for $\mathrm{D}$-arginine utilization as the sole source of carbon and nitrogen through L-arginine catabolic pathways in $P$. aeruginosa. In this study, enzymic properties of the catabolic FAD-dependent D-amino acid dehydrogenase DauA and the physiological functions of the dauBAR operon were further characterized with other D-amino acids. These results establish DauA as a D-amino acid dehydrogenase of broad substrate specificity, with D-Arg and D-Lys as the two most effective substrates, based on the kinetic parameters. In addition, expression of dauBAR is specifically induced by exogenous D-Arg and D-Lys, and mutations in the dauBAR operon affect utilization of these two amino acids alone. The function of DauR as a repressor in the control of the dauBAR operon was demonstrated by dauB promoter activity measurements in vivo and mobility shift assays with purified His-tagged protein in vitro. The potential effect of 2-ketoarginine (2-KA) derived from $\mathrm{D}$-Arg deamination by DauA as a signal molecule in dauBAR induction was first revealed by mutation analysis and further supported by its in vitro effect on alleviation of DauRDNA interactions. Through sequence analysis, putative DauR operators were identified and confirmed by mutation analysis. Induction of the dauBAR operon to the maximal level was found to require the L-arginine-responsive regulator $\mathrm{ArgR}$, as supported by the loss of inductive effect by LArg on dauBAR expression in the $\arg R$ mutant and binding of purified ArgR to the dauB regulatory region in vitro. In summary, this study establishes that optimal induction of the dauBAR operon requires relief of DauR repression by $2-K A$ and activation of ArgR by L-Arg as a result of D-Arg racemization by the encoded DauA and DauB.
\end{abstract}

\section{INTRODUCTION}

Although L-enantiomers of amino acids are selected by nature as building blocks of proteins, D-amino acids do exist in living organisms from bacteria to humans to serve a variety of specific functions. For example, D-amino acids are components in the synthesis of cell wall peptidoglycans and secondary metabolites (antibiotics, siderophores, etc.) in micro-organisms, and D-serine (D-Ser) plays an important role in the central nervous system of humans (Schell et al., 1995).

In living organisms, biosynthesis of free-form D-amino acids is catalysed by racemases with L-enantiomers as

Abbreviations: AST, arginine succinyltransferase; ATA, arginine transaminase; DADH, D-arginine dehydrogenase; INT, iodonitrotetrazolium chloride; 2-KA, 2-ketoarginine; PMS, phenazine methosulfate. substrates. Peptidyl D-amino acids arise either by taking free D-amino acid as substrate, as in cell wall synthesis (Vollmer et al., 2008), or by L-to-D epimerization, as in non-ribosomal peptide synthesis (Challis \& Naismith, 2004), in micro-organisms. In higher eukaryotic organisms, this process is infrequently catalysed by enzymedriven post-translational isomerization (Takahashi et al., 2008). Amino acid racemization can also occur at an accelerated rate by physical and/or chemical treatments. For example, racemization of L-lysine at elevated temperatures has great potential in the commercial process of $\mathrm{D}$ lysine (D-Lys) production (Takahashi et al., 1997).

Although D-amino acids are required for very specialized biological purposes, it is apparently not in the best interest of cells to maintain high concentrations of these rare amino acids. In the plant Arabidopsis thaliana, D-alanine (D-Ala) 
and D-Ser exert a toxic effect on growth, and introducing a recombinant D-amino acid oxidase to plants not only alleviates the toxic effect but also promotes growth (Erikson et al., 2004). In bacteria, D-Ala and D-glutamic acid (D-Glu), and in some cases D-Lys, are the natural ingredients of cell wall peptidoglycan. However, when they are provided in the growth medium, Escherichia coli is able to incorporate other D-amino acids into peptidoglycan, which results in severe alterations to cell wall structure (Caparros et al., 1992). Therefore, organisms with the capacity to degrade D-amino acids should have an advantage in surviving these potentially adverse challenges.

The biochemistry of D-amino acid catabolism has not been intensively studied in comparison with those of L-amino acids, and therefore information is still fragmentary. In general, D-amino acids are metabolized either directly or after conversion into the L-enantiomers. Many racemases from different organisms have been reported. It is the general notion that amino acid racemases serve in the biosynthesis of D-amino acids by taking L-isomers as substrates. In bacteria, alanine racemase and glutamate racemase are essential enzymes due to their functions in the biosynthesis of D-Ala and D-Glu, and hence in cell wall synthesis. Most amino acid racemases require pyridoxal 5' phosphate (PLP) as coenzyme, with a few exceptions. The structures and functions of the PLP-dependent and PLPindependent amino acid racemases have been clarified at the molecular level (Yoshimura \& Esak, 2003).

Our group recently reported a new type of D- to L-arginine racemization by coupled dehydrogenases (Fig. 1b): an FAD-dependent catabolic D-arginine (D-Arg) dehydrogenase DauA and a NADH/NADPH-dependent anabolic Larginine dehydrogenase $\mathrm{DauB}$ ( $\mathrm{Li} \& \mathrm{Lu}, 2009$ ). These two enzymes are encoded in the dauBAR operon, which is induced by exogenous D-Arg but not by L-Arg, and the conversion of D-Arg to L-Arg is the prerequisite for D-Arg utilization as the sole source of carbon and nitrogen through L-Arg catabolic pathways in Pseudomonas aeruginosa. This operon and its encoded enzymes are conserved among pseudomonads ( $\mathrm{Li} \& \mathrm{Lu}, 2009$ ) and many other related bacteria.

There are four catabolic pathways for L-Arg utilization in $P$. aeruginosa $(\mathrm{Lu}, 2006)$. The arginine decarboxylase pathway serves to supply putrescine, and hence spermidine, when arginine is abundant (Nakada \& Itoh, 2003). The arginine deiminase pathway provides ATP under anaerobic conditions (Vander Wauven et al., 1984), and its induction is mainly controlled by Anr, the homologue of E. coli Fnr, in response to oxygen status, as well as by ArgR, the L-Argresponsive regulator, as auxiliary factor in the presence of L-Arg. The arginine succinyltransferase (AST) pathway is the major route of L-Arg utilization as the carbon and nitrogen source under aerobic conditions (Jann et al., 1986), and its induction by L-Arg requires a functional ArgR (Fig. 1a). When the AST pathway is blocked, the arginine transaminase (ATA) pathway can still support cell growth on L-Arg, but with a longer generation time (Yang $\& \mathrm{Lu}, 2007)$. Induction of the ATA pathway is controlled by the AruSR two-component system (Fig. 1a), independent of ArgR. Mutants without AST and ATA pathways show no apparent growth on L-Arg as the sole source of carbon and nitrogen.

In this study, the enzymic properties of DauA, encoding a catabolic FAD-dependent D-amino acid dehydrogenase, and the physiological functions of the $d a u B A R$ genes were further characterized with a group of 19 D-amino acids. Regulation of the dauBAR operon was analysed by a combination of genetics and biochemical approaches with a variety of mutants and purified transcriptional regulators DauR and ArgR.

\section{METHODS}

Strains and growth conditions. Strains and plasmids used in the study are listed in Table 1. Luria-Bertani (LB) medium was used for strain construction with the following supplements as required: ampicillin at $100 \mu \mathrm{g} \mathrm{ml}^{-1}$ (E. coli); carbenicillin at $100 \mu \mathrm{g} \mathrm{ml}^{-1}(P$. aeruginosa). Minimal medium P (MMP) (Haas et al., 1977) was used for the growth of $P$. aeruginosa strains, with specific carbon $(\mathrm{C})$ and nitrogen (N) sources as indicated $(20 \mathrm{mM}$, unless specified otherwise).

Enzyme assays. DauA was purified as previously described ( $\mathrm{Li} \& \mathrm{Lu}$, 2009); the purified protein appeared as a mixture of monomer and dimer when analysed by SDS-PAGE under reducing conditions, which may be the result of non-covalent interactions mediated by the exposure of hydrophobic patches upon boiling (Shin et al., 2002). The substrate specificity of DauA was measured by D-amino acid dehydrogenase activity by a published method ( $\mathrm{Li} \& \mathrm{Lu}, 2009$ ). A total of $18 \mathrm{D}$-amino acids was used, including D-phenylalanine (DPhe), D-Lys, D-Glu, D-tryptophan (D-Trp), D-leucine (D-Leu), Dornithine (D-Orn), D-asparagine (D-Asn), D-histidine (D-His), D-tyrosine (D-Tyr), D-Ser, D-glutamine (D-Gln), D-proline (D-Pro), D-methionine (D-Met), D-threonine (D-Thr), D-valine (D-Val), Daspartic acid (D-Asp), D-Ala and D-Arg. D-Cysteine (D-Cys) was not included because the $-\mathrm{SH}$ group of $\mathrm{D}$-Cys causes reduction of iodonitrotetrazolium chloride (INT). The reaction mixture contained $100 \mathrm{mM}$ Tris/HCl (pH 8.7), $10 \mathrm{mM} \mathrm{KCN}, 0.2 \mathrm{mM}$ FAD, $0.8 \mathrm{mM}$ phenazine methosulfate (PMS), $0.8 \mathrm{mM}$ INT, $2.05 \mu \mathrm{g} \mathrm{DauA} \mathrm{ml} \mathrm{D}^{-1}$, and $20 \mathrm{mM}$ of the tested D-amino acid in a total volume of $0.5 \mathrm{ml}(\mathrm{Li}$ $\& \mathrm{Lu}, 2009)$. The reaction was initiated by the addition of DauA, and was continued for $3 \mathrm{~min}$ at $37{ }^{\circ} \mathrm{C}$ before being stopped by $4 \mathrm{M} \mathrm{HCl}$ $(50 \mu \mathrm{l})$. After addition of $5 \mu \mathrm{l}$ Triton $\mathrm{X}-100$ to dissolve the precipitates and appropriate dilution, $A_{500}$ readings were taken. The background reading with double-distilled $\mathrm{H}_{2} \mathrm{O}$ substitution of Damino acid in a parallel control reaction was subtracted from the readings with $\mathrm{D}$-amino acids and the specific activities were calculated thereafter. One unit of D-amino acid dehydrogenase activity was defined as the amount of enzyme that led to the reduction of one nanomole of INT per minute under the standard assay conditions.

After initial screening, seven good substrates (D-Arg, D-Lys, D-Phe, DMet, D-Orn, D-His and D-Tyr) were further analysed to determine the catalytic parameters. The assay was carried out with the same procedure as described above, except that different concentrations of $D$-amino acids were used. The final concentration of purified protein used in the reaction was $2.37 \mu \mathrm{g} \mathrm{ml}^{-1}$. The $K_{\mathrm{m}}, k_{\text {cat }}$ and $k_{\text {cat }} / K_{\mathrm{m}}$ values were determined by non-linear regression data analysis using SigmaPlot 9.0. 


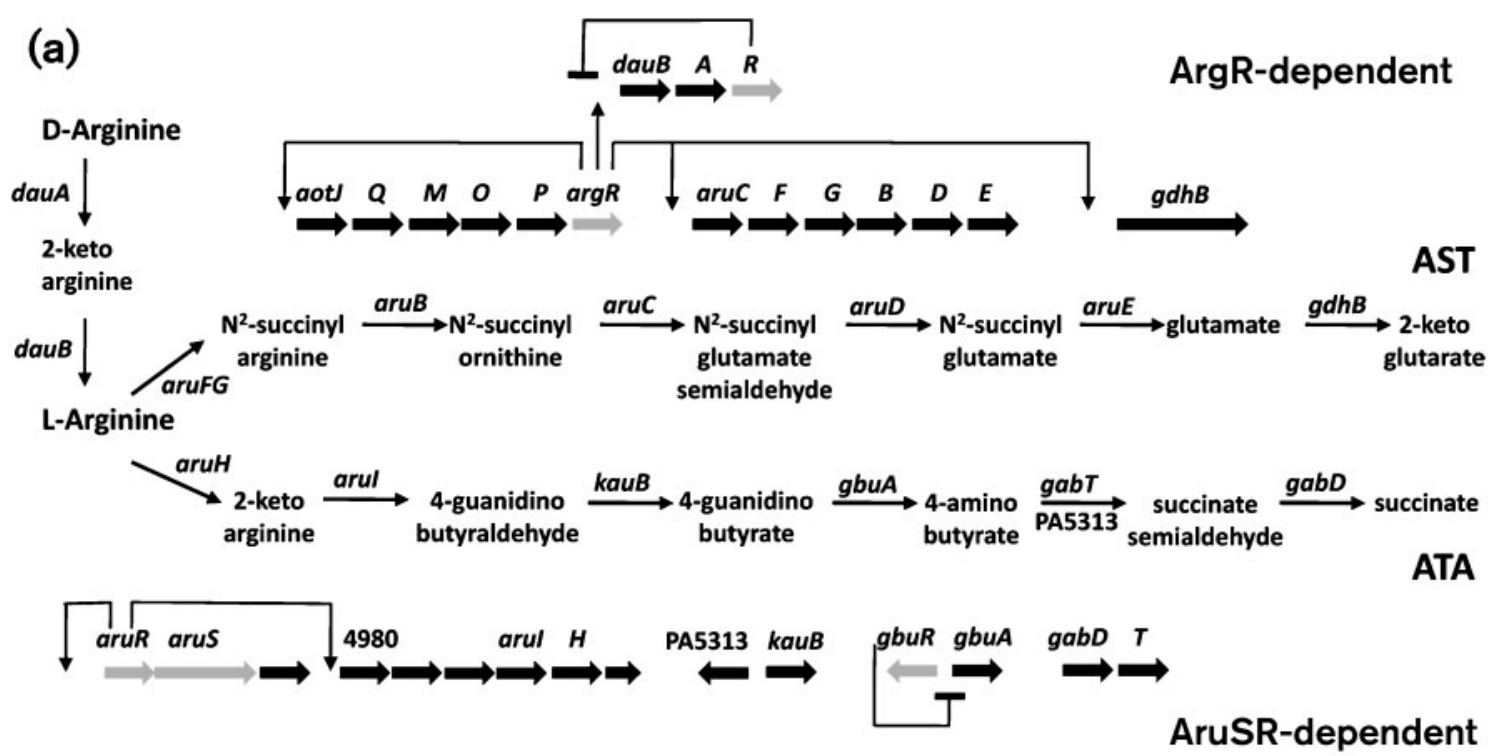

(b)

$$
\text { O" }
$$<smiles>[R]C(N)C(=O)OC</smiles>

D-Arginine D-Lysine

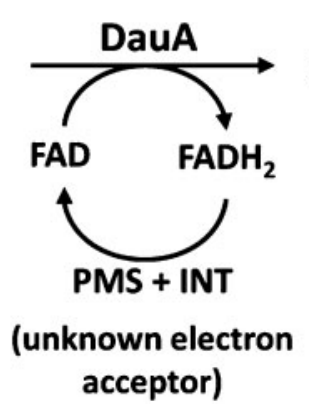

\section{4-guanidinobutyrate}<smiles>CC(C)COC(=O)O</smiles>

ketoarginine $+\mathrm{NH}_{3}$<smiles></smiles>

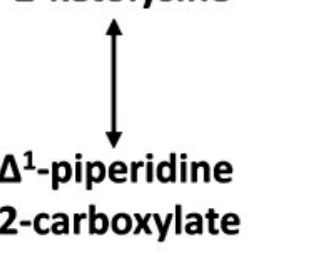

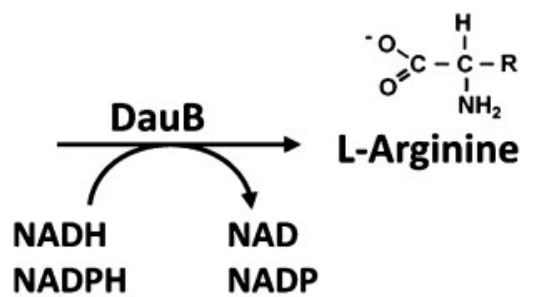

NADPH NADP

Fig. 1. Arginine catabolic pathways in $P$. aeruginosa PAO1. (a) Relevant intermediates of the ATA pathway, the AST pathway and D- to L-arginine racemization are shown in the biochemical reactions, and genes involved in these pathways are displayed as transcriptional units. The genes encoding transcriptional factors are marked as grey arrows, and promoters subject to activation and repression are labelled as arrowheads and solid bars, respectively. (b) Proposed functions of DauA and DauB in D-Arg and D-Lys catabolism. PMS and INT are artificial electron acceptors in the assays for DauA, as described in Methods, while the physiological electron acceptor remains unknown. Also shown are spontaneous chemical reactions of the keto acids derived from D-Arg and D-Lys.

Measurements of $\beta$-galactosidase activity were conducted in strains carrying plasmid pCR1 (dauB::lacZ) to analyse the effect of exogenous D-amino acids on the dauB promoter activity. Cells were grown at $37{ }^{\circ} \mathrm{C}$ in MMP supplemented with $20 \mathrm{mM} \mathrm{L}$-Glu in the absence or presence of $5 \mathrm{mM}$ D-amino acids, as indicated. Cells in the exponential phase were harvested by centrifugation and washed once with $20 \mathrm{mM}$ potassium phosphate buffer containing $1 \mathrm{mM}$ EDTA ( $\mathrm{pH}$ 7.6) and resuspended in the same buffer plus $1 \mathrm{mM}$ PMSF. Cells were broken with a French press at 17000 p.s.i. $(117300 \mathrm{kPa})$, and soluble cell extracts were prepared after centrifugation at $16000 \mathrm{~g}$ for
30 min. Protein concentration was determined by the Bradford method with BSA as standard (Bradford, 1976). For the measurements of $\beta$-galactosidase activity, ONPG was used as substrate (Miller, 1972).

Expression and purification of hexa-histidine-tagged DauR and ArgR. The structural genes dauR and $\arg R$ were amplified by PCR from the genomic DNA of strain PAO1 using the following primer pairs: 5' -AGC CCG AGC CAA GAC CCC AGC-3' and 5' -CCC AAG CTT CAG TCG CCG CCT TCC TTC-3' for dauR; 5' -TCC CCC GGG 
Table 1. Strains and plasmids used in this study

\begin{tabular}{|c|c|c|}
\hline Strain or plasmid & Genotype or description* & Source or reference \\
\hline \multicolumn{3}{|l|}{ E. coli strains } \\
\hline $\mathrm{DH} 5 \alpha$ & $\begin{array}{l}\mathrm{F} \phi 80 \mathrm{~d} l a c \Delta \mathrm{M} 15 \Delta(\text { lacZYA-argF }) \text { U169 deoR recA1 endA1 } \\
\left.\text { hsdR17( } \mathrm{r}_{\mathrm{K}} \mathrm{m}_{\mathrm{K}}\right) \text { supE44 } \lambda \text { thi-1 gyrA96 relA1 }\end{array}$ & Bethesda Research Laboratories \\
\hline TOP10 & 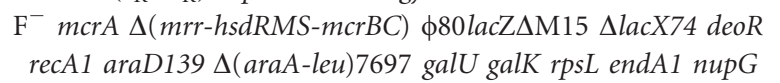 & Invitrogen \\
\hline \multicolumn{3}{|c|}{ P. aeruginosa strains } \\
\hline PAO5801 & dauB: $: \mathrm{Tc}^{\mathrm{r}}$ derivative of $\mathrm{PAO} 1$ & Nishijyo et al. (1998) \\
\hline PAO5802 & $\operatorname{dau} A:: \mathrm{Tc}^{\mathrm{r}}$ derivative of $\mathrm{PAO} 1$ & Haas et al. (1977) \\
\hline PAO5803 & dauR:: $\mathrm{Tc}^{\mathrm{r}}$ derivative of $\mathrm{PAO} 1$ & Li \& Lu (2009) \\
\hline PAO501 & $\operatorname{argR}:: \mathrm{Gm}^{\mathrm{r}}$ & Park et al. (1997) \\
\hline PAO5011 & dauB: : $\mathrm{Tc}^{\mathrm{r}}$ derivative of PAO501 & This study \\
\hline PAO5012 & dauA $:: \mathrm{Tc}^{\mathrm{r}}$ derivative of $\mathrm{PAO} 501$ & This study \\
\hline \multicolumn{3}{|l|}{ Plasmids } \\
\hline pRTP2 & $\begin{array}{l}\mathrm{Ap}^{\mathrm{r}} \mathrm{Sm}^{\mathrm{s}} \text { conjugation vector derived from } \mathrm{pRTP} 1 \text { with deletion } \\
\text { of EcoRI site }\end{array}$ & Li \& Lu (2009) \\
\hline pQF50 & bla lacZ transcriptional fusion vector & Li \& Lu (2009) \\
\hline pQF52 & bla lac $Z$ translational fusion vector & Li \& Lu (2009) \\
\hline pBAD-His6 & Vector modified from pBAD-HisA & Farinha \& Kropinski (1990) \\
\hline pCR1 & dauB: : lacZ transcriptional fusion of pQF50 & Park et al. (1997) \\
\hline pCR3 & For expression of His-tagged DauA & Li \& Lu (2009) \\
\hline pCR4 & For expression of His-tagged DauR & This study \\
\hline pCR5 & For expression of His-tagged ArgR & This study \\
\hline pZY1 & $g b u A::$ lacZ fusion of pQF52 & Li \& Lu (2009) \\
\hline
\end{tabular}

${ }^{\star} \mathrm{Tc}^{\mathrm{r}}$, tetracycline resistance; $\mathrm{Ap}^{\mathrm{r}}$, ampicillin resistance; $\mathrm{Sm}^{\mathrm{s}}$, streptomycin sensitivity.

ACT GCC CAA CCC CAA CGC ATC-3' and 5' -CCC AAG CTT CAG CCG CGT TCG ACG GGA GTG-3' for $\operatorname{argR}$. The resulting PCR products were digested with HindIII $($ dauR $)$ or SmaI plus HindIII $(\operatorname{argR})$, which cut at unique restriction sites introduced by the primers, and cloned into the SmaI and HindIII sites of the expression vector pBAD-His6 ( $\mathrm{Li} \& \mathrm{Lu}, 2009$ ). The resulting plasmids, designated pCR4 and pCR5, were introduced into E. coli TOP10.

For overexpression of DauR, E. coli TOP10 containing pCR4 was grown in LB medium containing ampicillin $\left(100 \mu \mathrm{g} \mathrm{ml}^{-1}\right)$ at $37{ }^{\circ} \mathrm{C}$ until the $\mathrm{OD}_{600}$ reached 0.5 , at which point $0.2 \%$ arabinose $(\mathrm{w} / \mathrm{v}$, final concentration) was added. After induction for $3 \mathrm{~h}$, the cells were harvested by centrifugation, resuspended in phosphate buffer (20 mM sodium phosphate, $500 \mathrm{mM} \mathrm{NaCl}, 20 \mathrm{mM}$ imidazole, $\mathrm{pH}$ 7.4) plus $1 \mathrm{mM}$ PMSF and ruptured by an Aminco French press at 17000 p.s.i. (117300 kPa). Cell debris was removed by centrifugation at $20000 \mathrm{~g}$ for $1 \mathrm{~h}$, and the resulting cell-free extract was further treated with streptomycin sulphate. After centrifugation, the resulting supernatant was applied to a HisTrap HP column (GE Healthcare) equilibrated with the above phosphate buffer. After washing off the unbound proteins, His-tagged DauR was eluted with a linear gradient of $20-500 \mathrm{mM}$ imidazole over 10 column volumes. Fractions containing DauR, as indicated by SDS-PAGE, were pooled, desalted by an Aminco Ultra-15 centrifugal filter unit (molecular mass cutoff $10 \mathrm{kDa}$, Millipore) and further purified by anion- exchange chromatography through a Mono Q HR5/5 column (GE Healthcare) equilibrated with $20 \mathrm{mM}$ Tris/ $\mathrm{HCl}$ ( $\mathrm{pH}$ 7.6; buffer A). The DauR protein was eluted by a linear gradient of $0-1 \mathrm{M} \mathrm{KCl}$ in buffer A over 20 column volumes. Target fractions that were homogeneous, as determined by visual inspection after SDS-PAGE, were pooled, desalted and concentrated using an Aminco Ultra-15 centrifugal filter unit (molecular mass cutoff $10 \mathrm{kDa}$, Millipore). The protein concentration in the final preparation was determined by the Bradford method with BSA as standard.

For overexpression of ArgR, E. coli TOP10 containing pCR5 was grown in LB medium containing ampicillin $\left(100 \mu \mathrm{g} \mathrm{ml}^{-1}\right)$ at $30{ }^{\circ} \mathrm{C}$. The steps described above were employed to prepare protein samples. After being applied to a HisTrap HP column (GE Healthcare), Histagged ArgR was eluted out at $300 \mathrm{mM}$ imidazole, as indicated by SDS-PAGE. Fractions with the desired purity were pooled and concentrated as described above.

Gel retardation analysis. The regulatory region of $d a u B$ was PCRamplified from plasmid pCR1 with the following primers: $5^{\prime}$-TAC AAG CTT CTG TAC AAC CTG GTG GCG CAG- ${ }^{\prime}$ and $5^{\prime}$-AAA CAA TTT GTC CAT TCT GGA CTT TAA GGT-3'. Probe dauB-Mu containing the same region with mutations on the predicted DauR operator site (from GTCCAN TGGAC to GTGGAN $_{3}$ TCCAC) was amplified with the same forward primer and the following reverse 
primer: 5'-AAA CAA TTT GTG GAT TCT CCA CTT TAA GGT-3'. The resulting PCR products of 602 bp were purified by gel extraction and used as probes in gel retardation analysis.

The binding reactions were carried out as follows. The $d a u B$ or $d a u B-$ $\mathrm{Mu}$ DNA probe was allowed to interact with different concentrations of DauR or ArgR in a mixture of $20 \mu$ containing $50 \mathrm{mM}$ Tris $/ \mathrm{HCl}$ $(\mathrm{pH} 7.5), 50 \mathrm{mM} \mathrm{KCl}, 1 \mathrm{mM}$ EDTA, $5 \%$ (v/v) glycerol, $200 \mu \mathrm{g}$ BSA ml ${ }^{-1}$ and $4 \mathrm{mM}$ DTT. When indicated, $10 \mathrm{mM}$ of specified compounds [2-ketoarginine (2-KA), L-Arg or D-Arg] was added to the reaction to analyse their potential effects on nucleoprotein complex formation. Reaction mixtures were incubated for $20 \mathrm{~min}$ at room temperature and an aliquot of $10 \mu \mathrm{l}$ was loaded on a $6 \%$ polyacrylamide gel while the gel was running. The gel was stained with SYBR Green I (1:10000 dilution, Invitrogen) for $20 \mathrm{~min}$, washed twice with deionized $\mathrm{H}_{2} \mathrm{O}$, and scanned by an Omega 10GD Imaging System (UltraLum) with a setting for excitation at $473 \mathrm{~nm}$ and emission at $520 \mathrm{~nm}$.

\section{RESULTS}

\section{Substrate specificity and catalytic parameters of DauA}

We have recently reported that purified DauA possesses a D-arginine dehydrogenase (DADH) activity ( $\mathrm{Li} \& \mathrm{Lu}$, 2009). Fig. 2 summarizes the substrate specificity of DauA. With a final concentration of $20 \mathrm{mM}$ in the reaction, several D-amino acids including D-Lys, D-Met, D-Phe, DTyr, D-Orn and D-His served as good substrates in addition to D-Arg. In fact, DauA even exhibited a higher activity towards D-Lys than towards D-Arg, which is due to a strong substrate inhibition effect of D-Arg at high concentrations as described below. In comparison, DauA showed very low or undetectable activity toward D-Thr, D-Asn, D-Glu, DGln, D-Ala, D-Asp and D-Ser.

The $k_{\text {cat }}, K_{\mathrm{m}}, K_{\mathrm{i}}$ (if applicable) and $k_{\text {cat }} / K_{\mathrm{m}}$ values of the seven good substrates as mentioned above were determined as shown in Table 2. It was apparent that $k_{\text {cat }} / K_{\mathrm{m}}$ values (Koshland, 2002) of D-Arg and D-Lys are far better than those of the other substrates. In addition, substrate inhibition was observed for D-Arg and D-Lys, with the former $\left(K_{\mathrm{i}}=24.1 \mathrm{mM}\right)$ exerting a much stronger effect than the latter $\left(K_{\mathrm{i}}=150.1 \mathrm{mM}\right)$. No substrate inhibition was observed for the other five D-amino acids.

\section{Effects of D-amino acids on the dauB promoter activity}

Since DauA possesses broad substrate specificity, the effect of exogenous D-amino acids on expression of the dauBAR operon was analysed in strain PAO1 carrying pCR1 (dauB::lacZ) grown overnight in glutamate minimal medium with or without D-amino acid supplements at $5 \mathrm{mM}$. Among the $19 \mathrm{D}$-amino acids tested, only D-Arg and D-Lys exerted 4.2-fold and 2.9-fold induction effects, respectively, while the other D-amino acids exhibited either no apparent effects or even inhibition effects (data not shown).

\section{Effects of dauA and dauB on D-amino acid utilization}

The physiological functions of $\operatorname{dau} A$ and $\operatorname{dauB}$ in D-amino acid utilization were analysed by comparing the growth phenotypes of PAO5801 (dauB) and PAO5802 (dauA) with

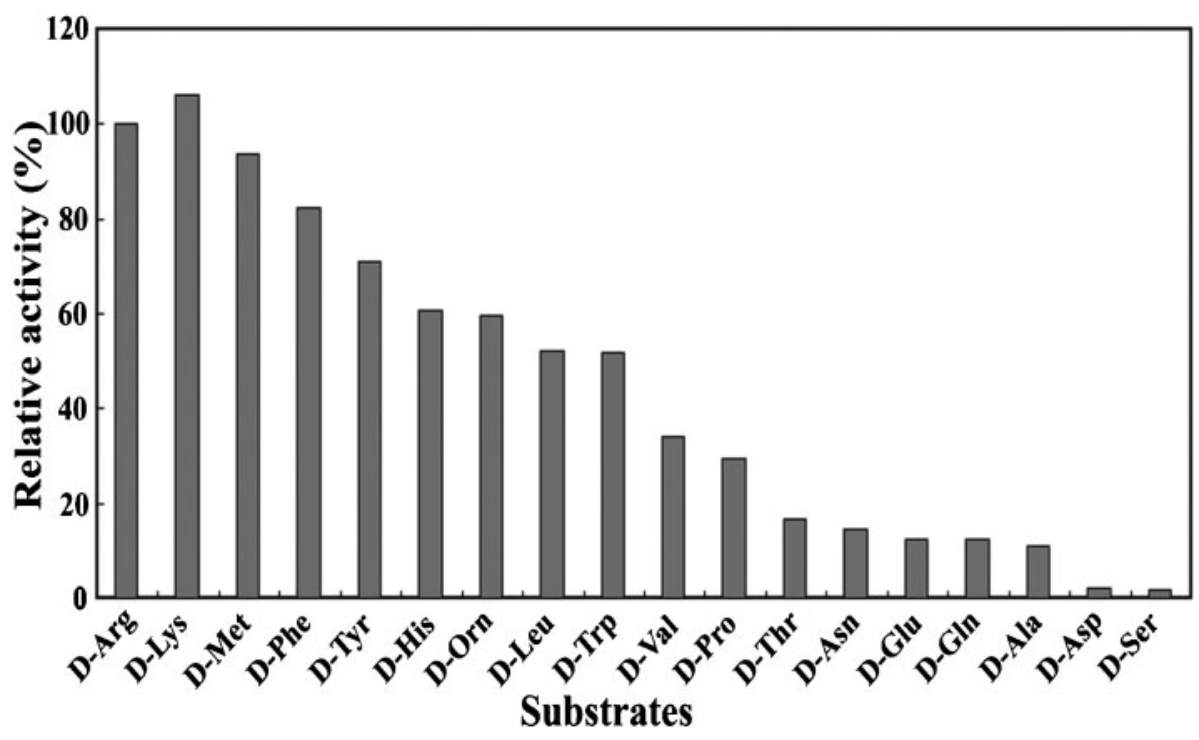

Fig. 2. Substrate specificity of DauA. The substrate specificity of DauA was determined from the D-amino acid dehydrogenase activity of the purified enzyme. The relative activity towards each D-amino acid was calculated with respect to the activity towards D-Arg, which was taken as $100 \%$. 
Table 2. Catalytic parameters of DauA

Values represent the average and range of two measurements.

\begin{tabular}{|lcccc|}
\hline Substrate & $\boldsymbol{k}_{\text {cat }}\left(\mathbf{s}^{-\mathbf{1}}\right)$ & $\boldsymbol{K}_{\mathrm{m}}(\mathbf{m M})$ & $\boldsymbol{k}_{\text {cat }} / \boldsymbol{K}_{\mathbf{m}}\left(\mathbf{M}^{-\mathbf{1}} \mathbf{s}^{-\mathbf{1}}\right)$ & $\boldsymbol{K}_{\mathbf{i}}(\mathbf{m M})$ \\
\hline D-Arginine & $11.1 \pm 0.7$ & $0.08 \pm 0.02$ & $1.5 \times 10^{5}$ & $24.1 \pm 7.1$ \\
D-Lysine & $9.2 \pm 0.3$ & $0.19 \pm 0.02$ & $4.8 \times 10^{4}$ & $150.1 \pm 53.0$ \\
D-Methionine & $6.8 \pm 0.3$ & $1.43 \pm 0.24$ & $4.8 \times 10^{3}$ & \\
D-Ornithine & $6.2 \pm 0.1$ & $1.48 \pm 0.13$ & $4.2 \times 10^{3}$ & \\
D-Phenylalanine & $5.3 \pm 0.2$ & $1.13 \pm 0.14$ & $9.7 \times 10^{3}$ & \\
D-Tyrosine & $3.6 \pm 0.1$ & $0.40 \pm 0.07$ & $1.0 \times 10^{3}$ & \\
D-Histidine & $3.6 \pm 0.1$ & $3.52 \pm 0.15$ & & \\
\hline
\end{tabular}

that of the parental strain PAO1 on D-amino acids, as listed in Table 3. PAO1 was able to grow on D-Arg/Lys and several other D-amino acids as sole nitrogen source, but only D-Arg/Glu/Gln/Ala can support its growth efficiently as the sole source of carbon and nitrogen. A lesion in $d a u A$ or $\operatorname{dauB}$ abolished utilization of D-Arg and D-Lys specifically. Without a functional $\operatorname{dauA}, \mathrm{PAO} 5802$ lost the capability to grow on these two D-amino acids as the sole nitrogen source. PAO5801 ( $\operatorname{dauB})$ grew similarly to the parental strain PAO1 on D-Arg or D-Lys as sole nitrogen source, but its growth on D-Arg as carbon source was abolished. Consistent with the induction effect of exogenous D-Arg and D-Lys on the $\operatorname{dauB}$ promoter, these results support the physiological functions of DauA and

Table 3. Growth phenotype of $\operatorname{dau} A$ and $\operatorname{dau} B$ mutants on D-amino acids

Cultures were inoculated with fresh cells with a starting $\mathrm{OD}_{600}$ of 0.02 , and growth was continued for 4 days before measurements of final $\mathrm{OD}_{600}$. ,$- \mathrm{OD}_{600}<0.1 ;+, 0.1 \leqslant \mathrm{OD}_{600}<0.2 ;++, 0.2 \leqslant \mathrm{OD}_{600}<0.6 ;+++, \mathrm{OD}_{600} \geqslant 0.6$.

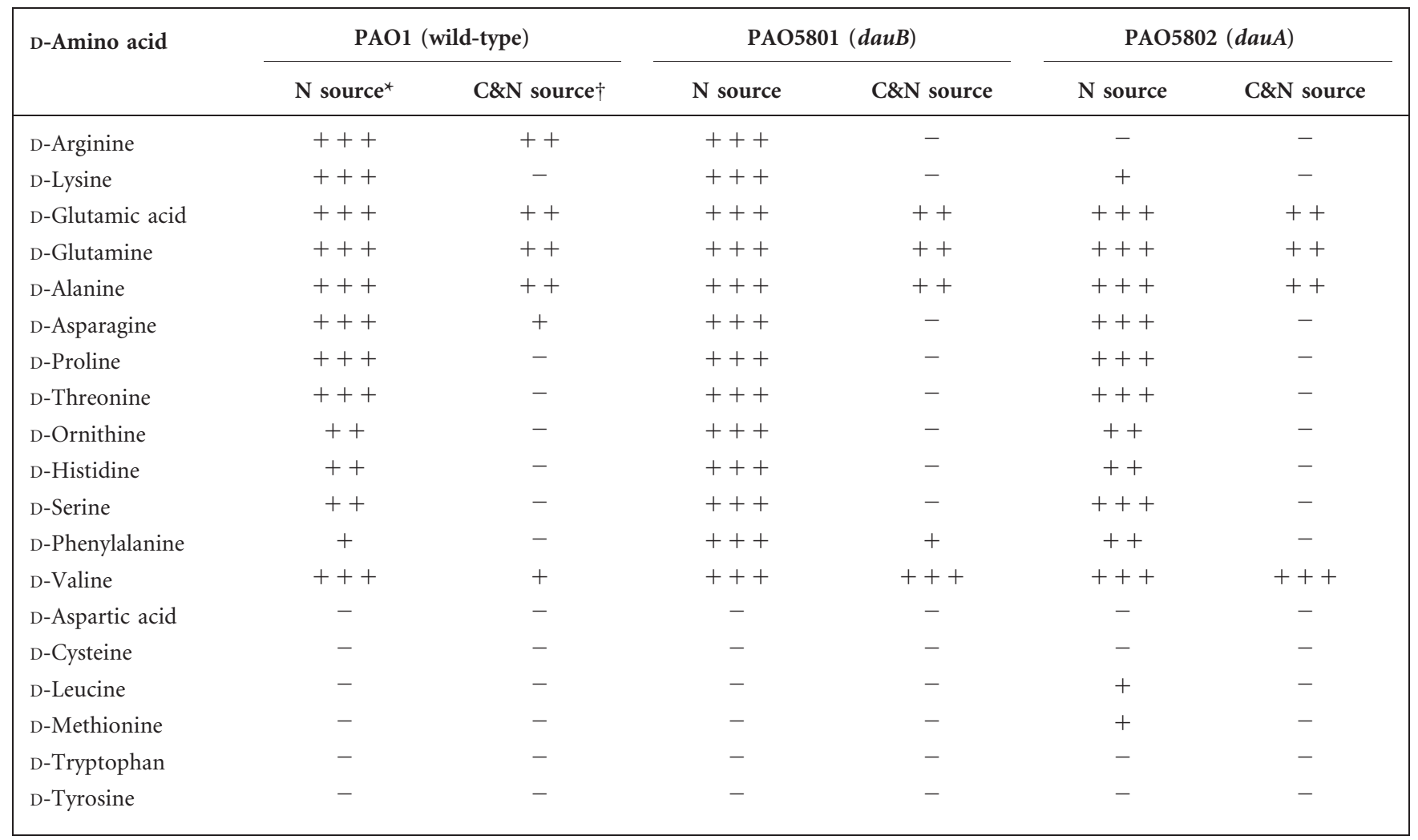

${ }^{*} \mathrm{~N}$ source: cells were grown in glucose $(20 \mathrm{mM})$ MMP with $5 \mathrm{mM} \mathrm{D}$-amino acids as nitrogen source.

$\dagger \mathrm{C} \& \mathrm{~N}$ source: cells were grown in MMP with $10 \mathrm{mM}$ D-amino acids ( $5 \mathrm{mM}$ for D-tyrosine) as carbon and nitrogen source. 
DauB on D-Arg and D-Lys catabolism. In contrast, the other five D-amino acids that were considered to be good substrates of DauA cannot induce the dauB promoter. Except for D-Arg and D-Lys, mutations in the dauBAR operon did not abolish utilization of the other D-amino acids tested in this study. Moreover, we noticed that utilization of D-Phe as nitrogen source and D-Val as carbon source was greatly enhanced in the $\operatorname{dau} A$ and $\operatorname{dauB}$ mutants. This suggests potential interactions of DauA/ DauB with enzymes yet to be identified for D-Phe/Val utilization.

\section{Growth complementation tests of arginine and lysine auxotrophs by D-Arg and D-Lys}

The physiological function of DauA and DauB in D-to-L racemization of arginine was supported by growth complementation of an arginine auxotroph (a lesion in $\operatorname{argB}$ ) by D-Arg in our previous report ( $\mathrm{Li} \& \mathrm{Lu}, 2009)$. Here we employed two more arginine auxotrophs (PAO0315 and PAO0362) and one arginine/lysine auxotroph (PAO0372) to test growth complementation by DArg and D-Lys. Consistent with the previous results, arginine auxotrophy can be complemented by either LArg or D-Arg ( $\mathrm{Li} \& \mathrm{Lu}, 2009$ ). In contrast, lysine auxotrophy can only be complemented by L-Lys but not D-Lys. Since exogenous D-Arg/Lys were both able to induce expression of dauBA as described above, the lack of growth complementation by D-Lys would therefore indicate the absence of D-to-L racemization of lysine through coupled DauA/DauB reactions.

\section{Effects of dauBAR on dauB promoter activity}

Expression of the dauB promoter was analysed by measurement of $\beta$-galactosidase activity in the dau mutants harbouring pCR1 (dauB::lacZ). As shown in Table 4, mutations of these dau genes exerted different effects on the $\operatorname{dauB}$ promoter activity in response to exogenous D-
Arg. In PAO1, the promoter activity and DADH activity were induced by 22.2 -fold and 27 -fold, respectively, when $5 \mathrm{mM}$ D-Arg was included in the growth medium. In the dauA mutant, the induction effect of D-Arg on the promoter activity was completely abolished. Surprisingly, in the $\operatorname{dauB}$ mutant the promoter was still inducible by DArg, but to a lesser extent, although the DADH level showed no induction. These results suggest that 2-KA, the catalytic product of DauA on D-Arg, but not D-Arg per se, serves as an internal signal of dauBAR induction by exogenous D-Arg. Consistent with the proposed regulatory function of DauR, the basal level of the dauB promoter activity in the dauR mutant was 5.7-fold higher than that in PAO1; however, exogenous D-Arg and L-Arg did further induce the promoter activity by 2.4 -fold and 3.2-fold, respectively. This pattern of promoter activity was also reflected in the levels of DADH activity: the basal level of DADH in the dauR mutant was 8.1-fold higher than that in PAO1, and exogenous D-Arg and L-Arg further induced promoter activity by about 2.8- and 3.3-fold, respectively. The induction effects of D-Arg and L-Arg on the promoter activity and DADH activity in dauR mutant suggested regulator(s) other than DauR in regulation of the dauBAR operon, and L-Arg (the racemization product of D-Arg by DauAB) might be the signal.

\section{Participation of ArgR in regulation of the dauBAR operon}

The potential effect of $\operatorname{ArgR}$ (the L-Arg-responsive regulator) in control of the dauBAR operon was analysed in dau mutants derived from PAO501 $(\operatorname{argR})$ and the results are shown in Table 4. In comparison with PAO1, the induction effect of D-Arg on promoter activity and DADH activity was much lower in the argR mutant, suggesting the involvement of $\mathrm{ArgR}$ in dauBAR regulation. Consistent with the prediction of L-Arg as the second signal compound in dauBAR expression, the induction effect of D-Arg and L-Arg in the dauR mutant was completely

Table 4. Effect of dauBAR and argR on regulation of dauB promoter activity

\begin{tabular}{|c|c|c|c|c|c|c|}
\hline \multirow[t]{2}{*}{ Strain (genotype) } & \multicolumn{3}{|c|}{ pCR1 $(\text { dauB: : lacZ })^{\star}$} & \multicolumn{3}{|c|}{$\mathrm{DADH}^{*}$} \\
\hline & Glu & Glu + D-Arg & Glu + L-Arg & Glu & Glu + D-Arg & Glu + L-Arg \\
\hline PAO1 (wild-type) & 35 & 776 & 68 & 49 & 1323 & 106 \\
\hline PAO5801 (dauB) & 35 & 233 & 149 & 27 & 35 & 36 \\
\hline PAO5802 (dauA) & 13 & 33 & 56 & $\mathrm{ND}$ & $\mathrm{ND}$ & ND \\
\hline PAO5803 (dauR) & 198 & 479 & 632 & 398 & 1097 & 1318 \\
\hline PAO501 $(\operatorname{argR})$ & 26 & 190 & 49 & 29 & 393 & 57 \\
\hline PAO5011 (dauB $\operatorname{argR})$ & 20 & 181 & 67 & 32 & 33 & 29 \\
\hline PAO5012 (dauA $\operatorname{argR})$ & 10 & 23 & 17 & ND & $\mathrm{ND}$ & $\mathrm{ND}$ \\
\hline PAO5013 (dauR $\operatorname{argR})$ & 150 & 163 & 192 & 290 & 328 & 342 \\
\hline
\end{tabular}

${ }^{\star}$ Specific activity $\left(\mathrm{nmol} \mathrm{min}{ }^{-1} \mathrm{mg}^{-1}\right.$ ): strains harbouring pCR1 were grown in MMP supplemented with $20 \mathrm{mM}$ Glu plus $5 \mathrm{mM}$ D-Arg or L-Arg. dauB promoter activity was determined with ONPG as the substrate and measurement of $A_{420}$. DADH activity was determined with D-Arg at $20 \mathrm{mM}$ as the substrate and measurement of $A_{500}$. ND, Not detectable. 
abolished in the $d a u R \arg R$ mutant. The function of L-Arg/ $\operatorname{ArgR}$ in $\operatorname{dau} B A R$ regulation was further supported by the observation that the promoter activity can be further induced in the $\operatorname{dauB}$ mutant (in which the D-to-L racemization is blocked) when $\mathrm{L}$-Arg is added to the growth medium in addition to D-Arg (233 versus

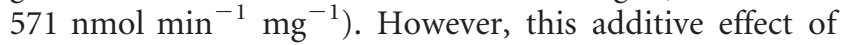
L-Arg did not exist in the $\operatorname{dauB}$ argR mutant (181 versus

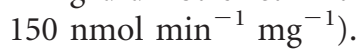

\section{Binding of DauR and ArgR to the regulatory region of dauB}

To further support the hypothesis of DauR and ArgR as transcriptional regulators of the $\operatorname{dau} B A R$ operon, gel retardation experiments were carried out with the purified recombinant His-tagged DauR or ArgR protein and DNA fragments carrying the regulatory region of dauB. As shown in Fig. 3(b), DauR interacts specifically with the regulatory region of $d a u B$, as evidenced by the formation of nucleoprotein complexes with slower mobility. When 2-KA at $10 \mathrm{mM}$ was added in the reaction, an inhibitory effect on nucleoprotein complex formation was observed, while addition of D-Arg or L-Arg showed no apparent effect. These results supported the predicted function of 2-KA as a signal compound for dauBAR expression through DauR.

Through genome comparison, a highly conserved palindromic sequence was identified in the $d a u B$ regulatory region of $P$. aeruginosa, Pseudomonas fluorescens and (a)

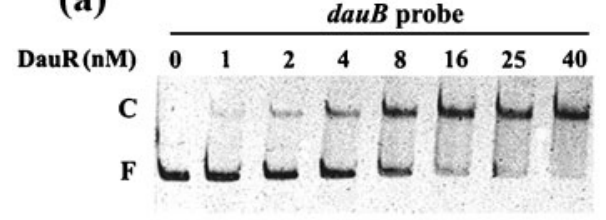

(b)

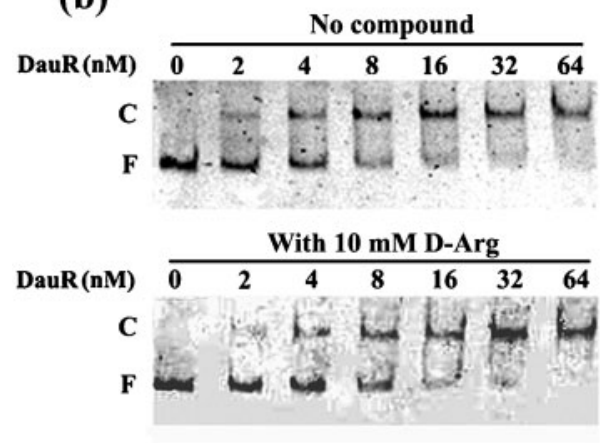

(c)

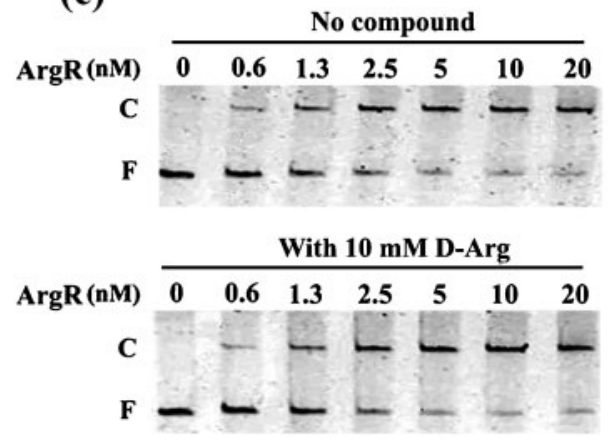

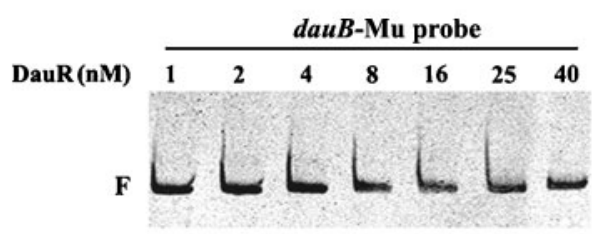

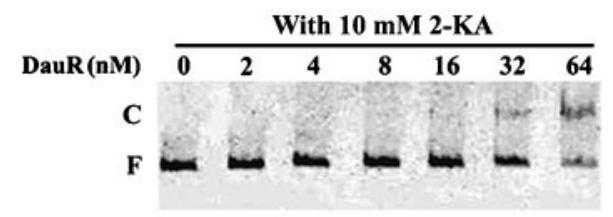

With 10 mM L-Arg

\begin{tabular}{llllllll}
$\operatorname{DauR}(\mathrm{nM})$ & 0 & 2 & 4 & 8 & 16 & 32 & 64 \\
\hline
\end{tabular}
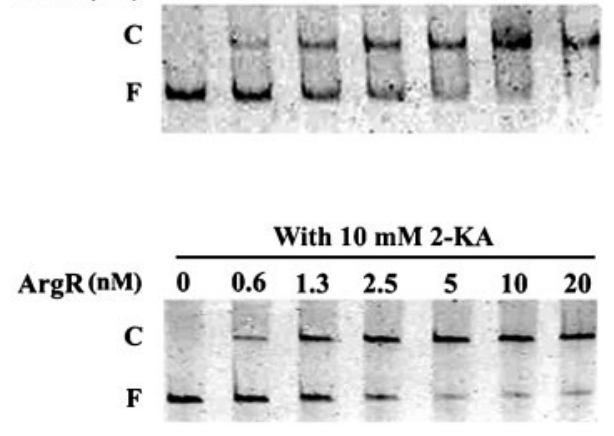

With 10 mM L-Arg

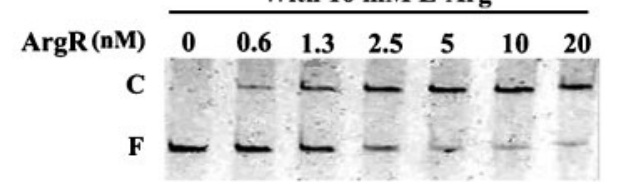

Fig. 3. Gel retardation assays of DauR and ArgR with the dauB regulatory region. (a) Electromobility shift analysis of $d a u B$ and dauB-Mu probes with recombinant His-tagged DauR. The dauB and dauB-Mu probes have identical sequences except for the DauR operator site (GTCCAGAATGGAC for probe dauB and GTGGAGAATCCAC for probe dauB-Mu). These probes at $0.27 \mathrm{nM}$ were titrated with DauR at the indicated concentrations. (b) Effect of different compounds on DauR-dauB interactions. The dauB probe at $0.13 \mathrm{nM}$ was added to the reaction at the indicated concentrations of DauR in the absence or presence of 2-KA, D-Arg and L-Arg at $10 \mathrm{mM}$. (c) Effect of different compounds on ArgR-dauB interactions. The dauB probe at $0.07 \mathrm{nM}$ was allowed to interact with ArgR at the indicated concentrations in the absence or presence of the same set of compounds as in panel (b). 
Pseudomonas mendocina (Fig. 4b). We propose this sequence to be the putative DauR operator site. To test this hypothesis, mutations on the proposed operator site in PAO1 were introduced, and this DNA probe (dauB-Mu of Fig. 3a) carrying the mutated operator site was unable to form a nucleoprotein complex with DauR.

The results of genetic studies strongly suggested that ArgR is a transcriptional activator of the dauBAR operon in response to the presence of L-Arg. In addition, sequence analysis of the $d a u B$ regulatory region identified a putative ArgR binding site (Fig. 4c). Binding of ArgR to the regulatory region of dauB was further demonstrated by electromobility shift analysis as shown in Fig. 3(c), and formation of ArgR-dependent nucleoprotein complex was not affected by the presence of 2-KA, L-Arg or D-Arg.

\section{Effects of D-Arg on the ATA pathway}

To serve as a carbon source, D-Arg needs to be converted into L-Arg through DauA and DauB (Li \& Lu, 2009) before being channelled into the AST or ATA pathway (Fig. 1). We have reported that genes of the AST pathway are induced by exogenous D-Arg, and this induction effect of D-Arg requires a complete D-to-L racemization. In contrast, D-Arg did not induce genes of the ATA pathway, except $g b u A$, encoding 4-guanidinobutyrase (Fig. 1a). To further substantiate this finding, the promoter activities of $g b u A$ and PA4980 (which drives transcription of aruH and aruI in the ATA pathway) were analysed in PAO1 and dau mutants in response to exogenous D-Arg and L-Arg.
As shown in Table 5, the promoter activity of PA4980 was significantly induced by exogenous L-Arg, while the effect of exogenous D-Arg was barely detectable in PAO1, and this pattern of expression was not affected by mutations in the dauBAR genes. In contrast, the gbuA promoter in PAO1 was induced by exogenous D-Arg but not by L-Arg, and this induction effect was completely abolished in the $\operatorname{dau} A$ mutant but sustained in the $\operatorname{dauB}$ mutant. These results were consistent with the previous observations with DNA microarrays (Li \& Lu, 2009), and their implications for the regulatory mechanisms of PA4980 and gbuA promoters are discussed below.

\section{DISCUSSION}

\section{The dauBAR operon is designated for D-Arg and D-Lys catabolism}

The dauBAR operon has been recently reported to participate in a unique D-to-L racemization of arginine by the coupled arginine dehydrogenases DauA and DauB (Li $\& \mathrm{Lu}, 2009)$. In this study, the enzymic properties of DauA, encoding a catabolic FAD-dependent D-amino acid dehydrogenase, and the physiological functions of the dauBAR genes were further characterized with a group of $19 \mathrm{D}-$ amino acids. The substrate specificity assay with the purified His-tagged DauA clearly demonstrated DauA to be a D-amino acid dehydrogenase of broad substrate specificity, with D-Arg and D-Lys being the two most (a)

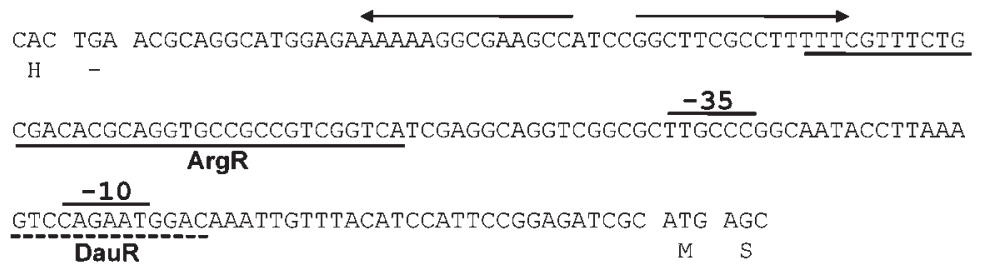

(b)

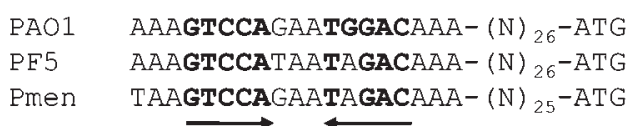

(c)

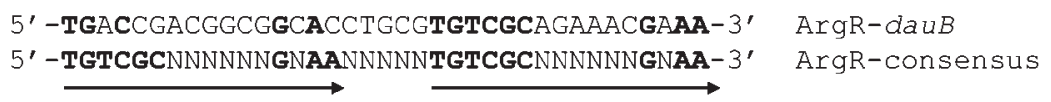

Fig. 4. ArgR and DauR binding sites in the dauB regulatory region. (a) Nucleotide sequence of the intergenic region between the ATG initiation codon of dauB and the TGA stop codon of its upstream $r h /$ gene. The proposed -10 and -35 regions of the dau $B$ promoter, the ArgR binding site and the DauR binding site are labelled. Also shown, by divergent arrows, is a putative rho-independent transcriptional terminator. (b) Multiple sequence alignment of DauR operators in the dau $B$ regulatory regions of pseudomonads. The putative DauR operator sites are highlighted, followed by the number of nucleotides before the ATG initiation codon of dauB. PAO1, P. aeruginosa PAO1; PF5, P. fluorescens Pf-5; Pmen, P. mendocina ymp. (c) Conservation of the ArgR binding site in the dau $B$ regulatory region. Nucleotides identical to those of the consensus sites are shown in bold type. The nucleotide sequence shown here corresponds to the complementary strand to that shown in panel (a). 
Table 5. Effect of L-Arg and D-Arg on PA4980 and gbuA promoter activity

\begin{tabular}{|c|c|c|c|c|c|c|}
\hline \multirow[t]{2}{*}{ Strain (genotype) } & \multicolumn{3}{|c|}{ pZY5 $($ PA4980:: lacZ)* } & \multicolumn{3}{|c|}{ pZY1 $(g b u A::$ lacZ)* } \\
\hline & Glu & Glu + D-Arg & Glu + L-Arg & Glu & Glu + D-Arg & Glu + L-Arg \\
\hline PAO5801 (dauB) & 2236 & 3607 & 13657 & 8 & 229 & 10 \\
\hline PAO5802 (dauA) & 2051 & 3628 & 15954 & 7 & 11 & 9 \\
\hline
\end{tabular}

${ }^{*}$ Strains harbouring pZY5 or pZY1 were grown in MMP supplemented with $20 \mathrm{mM}$ Glu plus $5 \mathrm{mM}$ D-Arg or $20 \mathrm{mM}$ L-Arg. The promoter activities were determined with ONPG as the substrate and measurement of $A_{420}$. The results [nmol $\mathrm{min}^{-1}$ (mg protein) ${ }^{-1}$ ] are the average of two measurements with SD lower than $5 \%$.

effective substrates based on the kinetic parameters (Fig. 2, Table 2). In addition, dauB promoter activity measurements with different $D$-amino acids suggested that expression of $d a u B A R$ is specifically induced by exogenous $\mathrm{D}$-Arg and D-Lys. The physiological functions of dauBAR in D-Arg and D-Lys catabolism were further supported by growth phenotype analysis, which demonstrated that mutations in $\operatorname{dauBAR}$ affect utilization of these two amino acids alone (Table 3 ). Therefore, we conclude that the dauBAR operon, which is conserved in pseudomonads, functions in D-Arg and D-Lys catabolism.

\section{D-Lys catabolism}

$P$. aeruginosa $\mathrm{PAO} 1$ growing on D-Lys as the sole nitrogen source requires DauA. This is supported by the fact that in the $\operatorname{dauA}$ mutant, utilization of D-Lys as the sole nitrogen source was almost abolished (Table 3). DauA catalyses oxidative deamination of D-Lys to ammonia and 2ketolysine (Fig. 1b). Non-enzymically, 2-ketolysine is in equilibrium with cyclic $\Delta^{1}$-piperidine-2-carboxylate predominantly, and possibly with other compounds (Friede \& Henderson, 1976; Kamio et al., 2009). While Pseudomonas putida has been proposed to possess enzymes for subsequent degradation of $\Delta^{1}$-piperidine-2-carboxylate in D-Lys catabolism as carbon source (Revelles et al., 2007), these enzymes seem to be missing in $P$. aeruginosa PAO1, as D-Lys cannot be used as sole carbon source in this organism. Moreover, since D-Lys does not complement the L-Lys requirement of a lysine auxotroph, it seems to have no D-to-L lysine racemization, either by coupled DauA/ DauB reactions or by the traditional racemases.

\section{D-Arg catabolism}

In $P$. aeruginosa $\mathrm{PAO} 1, \mathrm{D}-\mathrm{Arg}$ is capable of serving as the sole source of carbon and nitrogen. To be utilized as carbon source, D-Arg needs to be converted into L-Arg by DauA and DauB before being channelled into a complicated catabolic network, which is composed of several different pathways that are conserved among pseudomonads ( $\mathrm{Lu}, 2006)$. With a lesion in $\operatorname{dauA}$ or $\operatorname{dauB}$, the mutant strains failed completely to utilize D-Arg as the sole source of carbon (Table 3 ). D-to-L racemization of arginine is also evidenced by growth complementation of arginine auxotrophs with D-Arg.

Although racemization is also blocked in the dauB mutant, D-Arg is still able to serve as the sole source of nitrogen due to the release of ammonia from DauA-catalysed oxidative deamination; however, 2-KA as a second product from this reaction apparently cannot serve as the carbon source in a dauB mutant. This observation is somewhat contradictory to a report that PAO1 is able to grow on exogenous 2-KA as sole source of carbon and nitrogen (Jann et al., 1988). Perhaps this discrepancy may depend on the supply of 2KA. One possibility is that DauB facilitates electron transport of $\mathrm{FADH}_{2}$ in DauA and hence recycles the enzymic activity of DauA. Hence, in the dauB mutant, without a functional DauB to recycle the enzymic activity of DauA, the production of 2-KA is so low that it cannot support cell growth. The other possibility may relate to instability of 2-KA inside the cells. Like other keto acids, 2$\mathrm{KA}$ is liable to degrade into 4-guanidinobutyrate (4-GBA) in the presence of $\mathrm{H}_{2} \mathrm{O}_{2}$ ( $\mathrm{Li} \& \mathrm{Lu}, 2009$; Vlessis et al., 1990), which is abundant in actively growing cells under aerobic conditions. The latter hypothesis also explains another obscure observation that among genes of the ATA pathway for L-Arg catabolism, only $g b u A$, encoding guanidinobutyrase of the ATA pathway, was induced by D-Arg. It has been reported that 4-GBA serves as an inducer signal of gbuA (Nakada \& Itoh, 2002). As shown in Table 5, D-Arg per se does not induce $g b u A$; it is 2-KA, and most likely 4GBA derived from 2-KA degradation, that causes the observed induction.

\section{Why is D-Arg inefficient in the induction of the ATA pathway?}

We have reported that exogenous D-Arg and L-Arg are similar in induction of the aruCFGBDE operon of the AST pathway (Li \& Lu, 2009). In contrast, only exogenous LArg, but not D-Arg, was able to induce the PA4980 promoter, and hence aruHI of the ATA pathway (Table 5). Once transported into the cells, D-Arg is converted into LArg by DauA and DauB, but apparently the increment of LArg through this process has a dramatic discrepancy in induction of two L-Arg catabolic pathways. We considered 
that this discrepancy may be due to the nature of two regulatory mechanisms. For the aru operon (AST pathway), induction by L-Arg requires a functional $\operatorname{ArgR}$ (Park et al., 1997), a transcriptional regulator of the AraC family that most likely resides in the cytoplasm. For aruHI and the PA4980 promoter (ATA pathway), induction by L-Arg is controlled by the AruS/AruR two-component system (Yang \& Lu, 2007), with the sensor AruS anchored on the inner membrane and the response regulator AruR inside the cells, based on sequence analysis. Therefore, it is conceivable that the increment of intracellular L-Arg through D-Arg racemization inside the cells can be detected by ArgR but is completely missed by AruSR.

\section{Functions of DauR and ArgR on dauBAR regulation}

Expression of the dauBAR operon is subject to control by two transcriptional regulators, DauR as repressor and ArgR as activator. Positions of DauR and ArgR operator sites relative to the putative $\sigma^{70}$-dependent dauB promoter are shown in Fig. 4(a). Consistent with the proposed functions, the DauR binding site overlaps completely with the -10 region, and the ArgR binding site is located upstream of the -35 region. Primary sequence analysis revealed features of DauR as a transcriptional regulator. Specifically, the N terminus of DauR encloses an YheO-like PAS domain and the $\mathrm{C}$ terminus has a helix-turn-helix FIS domain which comprises a three-helix bundle motif, generally found in site-specific recombinases (Yang \& Steitz, 1995). Through multiple sequence alignment, the putative DauR operator sites were identified by genome comparison and confirmed by mutagenesis analysis in this study. The consensus DauR binding site (5'-GTCCAN 3 TGGAC-3') consists of two half-sites with a palindrome arrangement. The first halfsite sequences are relatively more conserved than the second half-site sequences.

In this study, we also established 2-KA, instead of D-Arg, to be the signal compound that interacts with DauR to alleviate its repression effect. Participation of 2-KA in control of dauBAR operon was supported by the finding that exogenous 2-KA or a dauR lesion increases the basal level of the dauB promoter (Table 4). In vitro, inclusion of 2-KA tends to inhibit the DauR-DNA interactions (Fig. $3 b)$. Once D-Arg was successfully converted into L-Arg by DauAB-dependent racemization inside the cells, the ArgR regulon was turned on in response to the elevated intracellular L-Arg pool, as revealed by transcriptome analysis (Li \& Lu, 2009).

ArgR, the arginine-responsive regulator protein, is autoinduced from the aot-argR operon for arginine uptake and regulation in $P$. aeruginosa (Nishijyo et al., 1998). ArgR has been found to participate in the regulation of a wide range of genes in arginine catabolic and anabolic metabolism. In the present study, the participation of $\operatorname{ArgR}$ in dauBAR operon regulation was demonstrated by dauB promoter activity measurement as well as electromobility shift analysis. The function of L-Arg as a signal compound for ArgR regulation was observed in the analysis of promoter activity. However, L-Arg did not affect the in vitro binding of ArgR to the dauB probe in the electromobility shift analysis, which has been shown to be the case for other ArgR operators in earlier studies (Park et al., 1997; Nishijyo et al., 1998). The other two compounds tested (D-Arg and 2-KA) also showed no effect on the binding of ArgR to the dauB operator. The ArgR protein of $P$. aeruginosa belongs to the AraC/XylS family of transcriptional regulators (Nishijyo et al., 1998), and therefore it is likely that binding of the signal ligand ( $\mathrm{L}-\mathrm{Arg}$ ) induces a conformational change of $\operatorname{ArgR}$ for its interactions with the RNA polymerase in promoter activation. In summary, this study established that optimal induction of the dauBAR operon requires relief of DauR repression by 2-KA and activation of ArgR by L-Arg as a result of D-Arg racemization by the encoded DauA and DauB arginine dehydrogenases.

\section{ACKNOWLEDGEMENTS}

We thank Dr Paul V. Phibbs from East Carolina University and the American Type Culture Collection for providing the PAO0315, PAO0362 and PAO0372 strains. The project was supported by National Science Foundation grant 0415608.

\section{REFERENCES}

Bradford, M. M. (1976). A rapid and sensitive method for the quantitation of microgram quantities of protein utilizing the principle of protein-dye binding. Anal Biochem 72, 248-254.

Caparros, M., Pisabarro, A. G. \& de Pedro, M. A. (1992). Effect of Damino acids on structure and synthesis of peptidoglycan in Escherichia coli. J Bacteriol 174, 5549-5559.

Challis, G. L. \& Naismith, J. H. (2004). Structural aspects of nonribosomal peptide biosynthesis. Curr Opin Struct Biol 14, 748-756.

Erikson, O., Hertzberg, M. \& Nasholm, T. (2004). A conditional marker gene allowing both positive and negative selection in plants. Nat Biotechnol 22, 455-458.

Farinha, M. A. \& Kropinski, A. M. (1990). Construction of broad-hostrange plasmid vectors for easy visible selection and analysis of promoters. J Bacteriol 172, 3496-3499.

Friede, J. D. \& Henderson, L. M. (1976). Metabolism of 5hydroxylysine in Pseudomonas fluorescens. J Bacteriol 127, 1239-1247.

Gallegos, M. T., Schleif, R., Bairoch, A., Hofmann, K. \& Ramos, J. L. (1997). Arac/XylS family of transcriptional regulators. Microbiol Mol Biol Rev 61, 393-410.

Haas, D., Holloway, B. W., Schambock, A. \& Leisinger, T. (1977). The genetic organization of arginine biosynthesis in Pseudomonas aeruginosa. Mol Gen Genet 154, 7-22.

Jann, A., Stalon, V., Wauven, C. V., Leisinger, T. \& Haas, D. (1986). $N^{2}$-Succinylated intermediates in an arginine catabolic pathway of Pseudomonas aeruginosa. Proc Natl Acad Sci U S A 83, 4937-4941.

Jann, A., Matsumoto, H. \& Haas, D. (1988). The fourth arginine catabolic pathway of Pseudomonas aeruginosa. J Gen Microbiol 134, 1043-1053.

Kamio, M., Ko, K. C., Zheng, S., Wang, B., Collins, S. L., Gadda, G., Tai, P. C. \& Derby, C. D. (2009). The chemistry of escapin: identification and quantification of the components in the complex 
mixture generated by an L-amino acid oxidase in the defensive secretion of the sea snail Aplysia californica. Chemistry 15, 1597-1603.

Koshland, D. E. (2002). The application and usefulness of the ratio $k_{\text {cat }} / K_{\mathrm{M}}$. Bioorg Chem 30, 211-213.

Li, C. \& Lu, C. D. (2009). Arginine racemization by coupled catabolic and anabolic dehydrogenases. Proc Natl Acad Sci U S A 106, 906911.

Lu, C. D. (2006). Pathways and regulation of bacterial arginine metabolism and perspectives for obtaining arginine overproducing strains. Appl Microbiol Biotechnol 70, 261-272.

Miller, J. H. (1972). Experiments in Molecular Genetics. Cold Spring Harbor, NY: Cold Spring Harbor Laboratory.

Nakada, Y. \& Itoh, Y. (2002). Characterization and regulation of the gbuA gene, encoding guanidinobutyrase in the arginine dehydrogenase pathway of Pseudomonas aeruginosa PAO1. J Bacteriol 184, 33773384 .

Nakada, Y. \& Itoh, Y. (2003). Identification of the putrescine biosynthetic genes in Pseudomonas aeruginosa and characterization of agmatine deiminase and $\mathrm{N}$-carbamoylputrescine amidohydrolase of the arginine decarboxylase pathway. Microbiology 149, 707714 .

Nishijyo, T., Park, S. M., Lu, C. D., Itoh, Y. \& Abdelal, A. T. (1998). Molecular characterization and regulation of an operon encoding a system for transport of arginine and ornithine and the ArgR regulatory protein in Pseudomonas aeruginosa. J Bacteriol 180, 5559-5566.

Park, S. M., Lu, C. D. \& Abdelal, A. T. (1997). Cloning and characterization of $\arg R$, a gene that participates in regulation of arginine biosynthesis and catabolism in Pseudomonas aeruginosa PAO1. J Bacteriol 179, 5300-5308.

Revelles, O., Wittich, R. M. \& Ramos, J. L. (2007). Identification of the initial steps in D-lysine catabolism in Pseudomonas putida. J Bacteriol 189, 2787-2792.
Schell, M. J., Molliver, M. E. \& Snyder, S. H. (1995). D-Serine, an endogenous synaptic modulator: localization to astrocytes and glutamate-stimulated release. Proc Natl Acad Sci U S A 92, 3948-3952.

Shin, I., Wachtel, E., Roth, E., Bon, C., Silman, I. \& Weiner, L. (2002). Thermal denaturation of Bungarus fasciatus acetylcholinesterase: is aggregation a driving force in protein unfolding? Protein Sci 11, 20222032.

Takahashi, E., Furui, M., Seko, H. \& Shibatani, T. (1997). D-Lysine production from L-lysine by successive chemical racemization and microbial asymmetric degradation. Appl Microbiol Biotechnol 47, 347351.

Takahashi, K., Uchida, C., Shin, R. W., Shimazaki, K. \& Uchida, T. (2008). Prolyl isomerase, Pin1: new findings of post-translational modifications and physiological substrates in cancer, asthma and Alzheimer's disease. Cell Mol Life Sci 65, 359-375.

Vander Wauven, C., Pierard, A., Kley-Raymann, M. \& Haas, D. (1984). Pseudomonas aeruginosa mutants affected in anaerobic growth on arginine: evidence for a four-gene cluster encoding the arginine deiminase pathway. J Bacteriol 160, 928-934.

Vlessis, A. A., Bartos, D. \& Trunkey, D. (1990). Importance of spontaneous $\alpha$-ketoacid decarboxylation in experiments involving peroxide. Biochem Biophys Res Commun 170, 1281-1287.

Vollmer, W., Blanot, D. \& de Pedro, M. A. (2008). Peptidoglycan structure and architecture. FEMS Microbiol Rev 32, 149-167.

Yang, Z. \& Lu, C. D. (2007). Functional genomics enables identification of genes of the arginine transaminase pathway in Pseudomonas aeruginosa. J Bacteriol 189, 3945-3953.

Yang, W. \& Steitz, T. A. (1995). Crystal structure of the site-specific recombinase $\gamma \delta$ resolvase complexed with a 34 bp cleavage site. Cell 82, 193-207.

Yoshimura, T. \& Esak, N. (2003). Amino acid racemases: functions and mechanisms. J Biosci Bioeng 96, 103-109.

Edited by: W. Quax 